\title{
Abstracts of 20th Annual ASENT Meeting
}

Published online: 9 July 2018

(C) The American Society for Experimental NeuroTherapeutics, Inc. 2018
Title: A Neurometabolic Approach to Treating Alzheimer's Disease: Hypothesis Testing in a Phase 2a Exploratory Clinical Trial with the New Chemical Entity T3D-959

Authors: John Didsbury, PhD, Hoda Gabriel, Warren Strittmatter, MD, Stan Chamberlain, $\mathrm{PhD}$

T3D Therapeutics, Inc.

\begin{abstract}
A major test of the neurometabolic hypothesis of Alzheimer's disease (AD) pathophysiology [i.e. dysfunctional glucose and lipid metabolism being key drivers of disease pathologies] relied upon a phase 3 clinical study of the PPAR gamma-selective agonist rosiglitazone. This trial failed to demonstrate statistically significant improvements in cognitive and functional tests and factored into the pursuit of other approaches to $\mathrm{AD}$ drug development, in particular, preventing or reversing structural events, beta-amyloid plaques and tau tangles. We contend that the neurometabolic hypothesis cannot be appropriately tested with this agent (or with pioglitazone). Restricted PPAR gamma target expression in the brain, poor blood-brain barrier penetration and high metabolism of rosiglitazone prevents bona fide hypothesis testing. The need exists to test the neurometabolic hypothesis with an agent superior to rosiglitazone. T3D-959 is an investigational new drug product in phase 2 clinical development as a potential disease-remedial therapy to slow, stop or reverse the course of AD. This chemical compound is orally delivered and administered once a day. T3D-959 is the first PPAR delta-activating compound (agonist) to be developed for the treatment of AD. Uniquely, this drug also activates PPAR gamma (dual agonist) at 15-fold lower potency, which may provide potential additive or synergistic effects in regulating dysfunctional brain glucose and lipid metabolism in AD. Numerous attributes of this agent make it a superior choice to rosiglitazone (and pioglitazone) to truly test the neurometabolic hypothesis. These include good penetration of the blood-brain barrier with indirect clinical evidence of cerebral target engagement, a drug metabolism profile which, in pre-clinical and phase 1 clinical studies, demonstrates a high likelihood of achieving multiples of its EC50 in the brain, from an oral dose, a good therapeutic
\end{abstract}

index and, ubiquitous high brain expression of the primary PPAR delta drug target. An exploratory/feasibility phase 2a clinical trial [ClinicalTrials.gov (NCT02560753)] in 36 subjects with mild-to-moderate AD (MMSE $=14-26$, average $=19.9)$ has been completed. The purpose of the phase 2a clinical study was to demonstrate that T3D-959 could produce desired changes in cerebral glucose metabolism (primary outcome measure - FDG-PET) that may indicate potential for cognitive improvement (secondary/ exploratory measures ADAS- $\operatorname{cog} 11$ and DSST). The therapeutic approach was based on the hypothesis that correcting insulin resistance in the brain (highly correlated with $\mathrm{AD}$ and potential key driver of $\mathrm{AD}$ pathophysiology) may be disease remedial. The study was a multi-center (3), randomized, parallel, four-dose design in subjects with mild-to-moderate Alzheimer's disease. Thirty-six subjects were randomized to one of four doses of T3D-959 (3, 10, 30 or $90 \mathrm{mg}$ ). T3D-959 was taken once daily for 14 days. Subjects were evaluated for changes from baseline in relative cerebral metabolic rate of glucose (FDG-PET imaging), functional connectivity (BOLD-fMRI), cognitive function (ADAS-Cog11 and DSST) and plasma metabolomics, as well as assessed for safety and tolerability to T3D959. In this study, plasma drug levels and metabolic analysis support typical systemic PPAR delta and gamma pharmacology. Exploratory FDG-PET neuroimaging outcomes indirectly support dose-dependent brain penetration by T3D-959, and directly demonstrate improvements in relative cerebral rates of glucose metabolism in multiple brain regions. Comparisons to rosiglitazone indicate this agent to be a superior molecule for assessing the contributions of dysfunctional metabolism to AD pathogenesis. This study was supported in part by grant AG-049510 from the NIH.

Title: CT1812 Exhibits a Synaptoprotective Effect in Alzheimer's Disease Patients

Authors: Susan M. Catalano, $\mathrm{PhD}^{1}$, Lon S Schneider, MD, $\mathrm{MS}^{2}$; Steven DeKosky, $\mathrm{MD}^{3}$; Roger Morgan, $\mathrm{MD}^{4}$, Courtney 
Rehak $^{1}$, Colleen Silky ${ }^{1}$, Kelsie Mozzoni ${ }^{1}$, Nicholas J Izzo, $\mathrm{PhD}^{1}$, Michael Grundman, MD, MPH ${ }^{1,5}$, Michael Schirm, $\mathrm{PhD}^{6}$, Rudolf Guilbaud, $\mathrm{MSc}^{6}$, Mark Watson, $\mathrm{PhD}^{6}$, Daniel Chelsky, $\mathrm{PhD}^{6}$, Charles Davis $\mathrm{PhD}^{7}$, Henrik Zetterberg, MD, $\mathrm{PhD}^{8}$, Kaj Blennow, MD, $\mathrm{PhD}^{8}$.

${ }^{1}$ Cognition Therapeutics, Pittsburgh, PA; ${ }^{2}$ Keck School of Medicine of USC, Los Angeles, CA; ${ }^{3}$ McKnight Brain Institute, University of Florida, Gainesville, FL; ${ }^{4}$ MedSurgPI, LLC Raleigh, North Carolina; ${ }^{5}$ Global R\&D Partners, LLC, San Diego, California; ${ }^{6}$ Caprion Biosciences, Inc., Montreal, Canada; ${ }^{7} \mathrm{CSD}$ Biostatistics, Inc., Arizona; ${ }^{8}$ University of Gothenburg, Sweden

Abstracts: CT1812 shows evidence of synaptic protection by displacing $\mathrm{A}^{2}$ oligomers from synaptic receptor sites, clearing oligomers from brain into CSF, and restoring synapse number and cognitive performance in aged transgenic mouse models of Alzheimer's disease (AD). Cognitive impairment in AD correlates most closely with synapse number. Neurogranin (NRGN) and synaptotagmin-1 (SYT-1) are important for synaptic function; CSF concentrations are elevated 27 and 52\%, respectively, in $\mathrm{AD}$ patients compared to controls as a result of CNS synaptic damage. We measured concentrations of these synaptic damage biomarkers in CSF samples from mild-tomoderate Alzheimer's patients (MMSE 18-26) at baseline and after 28 days of CT1812 treatment. A double-blind, placebo-controlled parallel group trial was performed with three doses of CT1812 (90, 280 and $560 \mathrm{mg})$ or placebo ( $N=4$ or 5 patients/group) given once daily. At 28 days, placebo-treated patients' CSF NRGN and SYT-1 increased 11 and $24 \%$ vs. their own baseline, while CT1812-treated patients' CSF NRGN decreased by 18 and 19\% respectively. The differences between the pooled CT1812 dose groups vs. placebo was significant for NRGN $(p=0.050)$ and for SYT-1 $(p=0.011)$. The reductions of NRGN and SYT-1 are consistent with a positive effect on synapses in Alzheimer's patients and with CT1812's mechanism of action. Further trials will examine the concentrations of these synaptic markers following longer term administration of CT1812.

Title: GM6, an Endogenous Multiple-Target Regulator that Provides a Novel Therapeutic Strategy for Treatment of ALS and Other Neurodegenerative Diseases

Authors: Mark Kindy ${ }^{1,2}$, William R. Swindell ${ }^{3}$, Krzysztof Bojanowski ${ }^{4}$, Paul Lupinacci ${ }^{5}$, Robert Bowser ${ }^{6}$, Raphael $\mathrm{Nir}^{7}$, Marisa Westlin ${ }^{7}$, James Evans, ${ }^{8}$ Tony Shum ${ }^{9}$, Raymond Chau ${ }^{9}$, Dorothy Ko 9 .

${ }^{1}$ Department of Pharmaceutical Sciences, College of Pharmacy, University of South Florida, Tampa, FL, USA. ${ }^{2}$ James A. Haley VAMC, Tampa, FL, USA. ${ }^{3}$ Ohio University, Heritage College of Osteopathic Medicine, Athens, OH, USA. ${ }^{4}$ Sunny BioDiscovery, Inc., Santa Paula,
CA, USA. ${ }^{5}$ Villanova University, Villanova, PA, USA. ${ }^{6}$ Iron Horse Diagnostics Inc., Scottsdale, AZ, USA. ${ }^{7}$ SBH Sciences, Natick, MA. ${ }^{8}$ Phenovista Biosciences, San Diego, CA. ${ }^{9}$ Genervon Biopharmaceuticals, LLC, Pasadena, CA, USA.

Abstract: Genervon Biopharmaceuticals hypothesized that the traditional single-target approach to treating neurodegenerative diseases may not be effective due to the complexity of these conditions and their multifactorial pathogenesis. Using a systems biology mindset, Genervon has searched for, discovered, and developed GM6 (aka GM604 in ALS trial), a 6amino acid peptide drug, modeled upon an endogenous embryonic stage motor neuronotrophic factor (MNTF) isolated from the developing nervous system and has multi-target regulatory effect. The therapeutic potential of GM6 was studied by in vitro studies, in vivo studies, and human clinical studies. In vitro assays using rat cortical neurons demonstrated that GM6 protected against the toxic factors of cerebral spinal fluid (CSF) from post-mortem CNS disease patients and increased cell survival $(p<0.0001)$. The resulting neuron survival as compared with untreated neurons increased to the following: ALS (175\%), Multiple Sclerosis (246\%), Alzheimer's Disease (191\%), Huntington's Disease (273\%) and Parkinson's Disease (198\%). Through in vivo studies we have shown that GM6, a cationic peptide, can pass through the blood-brain barrier and transit to the brain. We have also shown efficacy of GM6 in animal models for ALS (SOD1 mice), Parkinson's Disease (MPTP, 6-OHDA), and Multiple Sclerosis (EAE). GM6 prevents functional decline and extends survival. Genervon's phase 1 clinical trial with healthy subjects demonstrated GM6 has no clinically significant adverse events as adverse events were similar between treated and placebo groups. Our pilot ALS phase 2A clinical trial was conducted at Columbia Presbyterian Hospital and Massachusetts General Hospital. The protocol consisted of six intravenous injections of GM6 administered over 2 weeks and evaluated over 12 weeks. GM6 was shown to be safe and tolerable. The trial data suggested a positive signal in the clinical outcome of GM6 treated ALS patients. We observed significant clinical trial site-specific improvement in forced vital capacity (FVC). GM6 favorably downshifts the plasma levels in TDP-43, Tau and SOD1 biomarkers of ALS disease. Genervon's Parkinson's Disease phase 2A clinical trial conducted IV administration over 2 weeks and showed that GM6 is safe and well tolerated. The GM6-treated group had modest increase in plasma BDNF with statistical significance when compared to placebo patients at the end of dosing. DNA Microarray and RNA-seq were used to determine whether CNS diseaseassociated genes exhibit unique responses to GM6 treatment in SH-SY5Y cells, which would suggest that GM6 is impacting signaling pathways linked to processes that underlie CNS disease onset and/or progression. Bioinformatic analyses identified GM6-regulated genes with potentially 
important roles in the development and progression of multiple CNS diseases. These genes were categorized with respect to multiple hypothesized mechanisms of action which are also related to the pathology of multiple neurodegenerative diseases such as Parkinson's Disease, Alzheimer's Disease, Multiple Sclerosis, and Huntington's Disease. GM6 is a phase 3 clinical trial ready for ALS and a phase 2 clinical trial ready for Parkinson's Disease, Alzheimer's Disease, Multiple Sclerosis, and Huntington's Disease. Genervon is now inviting Pharmaceutical partners to help advance GM6 from a development-stage drug candidate to a marketable therapy for the many victims of neurodegenerative diseases and disorders that desperately need help.

Title: Secretory Products of B Cells from Blood of Multiple Sclerosis Patients Are Toxic to Oligodendrocytes and Neurons: Characterization of Toxic Factors and Use of Bioassay as a Screen of Potential Protective Therapies

Authors: Robert P Lisak, MD ${ }^{1,2}$, Liljana Nedelkoska ${ }^{1}$, Hanane Touil ${ }^{3}$, Paul M Stemmer ${ }^{4}$, Nicholas J Carruthers ${ }^{4}$, Amit Bar-Or ${ }^{3}$, Joyce A Benjamins 1,2

${ }^{1}$ Department of Neurology, Wayne State University School of Medicine, Detroit, MI, USA; ${ }^{2}$ Department of Microbiology, Immunology and Biochemistry, Wayne State University School of Medicine, Detroit, MI, USA; ${ }^{3}$ Center for Neuroinflammation and Neurotherapeutics, Department of Neurology, Perelman School of Medicine, University of Pennsylvania, Philadelphia, PA; ${ }^{4}$ Institute of Environmental Health Sciences, Wayne State University, Detroit, MI, USA.

\begin{abstract}
We reported that supernatants (Sup) from cultures of blood B cells from patients with multiple sclerosis (MS) are the cause death of rat oligodendrocytes (OL) and rat and human neurons in vitro. Sup from B cells of normal controls (NC) have little or no such effect. Cell death is independent of immunoglobulin (Ig) concentration, complement activity or levels of 37 cytokines and related proteins. Apoptosis, as measured by three assays, appears to be a major mechanism of cell death for both OL and neurons. We have suggested that secretory products of $\mathrm{B}$ cells in the meninges may mediate demyelination/OL death and neuronal/axonal toxicity in the underlying cortical gray matter. To characterize the factors/s inducing death of OL and neurons, using filters of different pore size, the factor/s for OL and neurons was/were in the > $300 \mathrm{kDa}$ fraction of the Sup. Using either an exosome isolation kit or ultracentrifugation at $100,000 \times g$ (UC) of the $>$ $300 \mathrm{kDa}$ fraction, toxicity in the MS B cell Sup appears to be in or associated with microvesicles, likely exosomeenriched (Ex-En), with no evidence of toxicity in similar preparations from NC. We are currently characterizing the size and appearance of these fractions as well as protein and lipid compositions. Preliminary proteomic analysis of UC preparations
\end{abstract}

from the $>300 \mathrm{kDa}$ fraction of Sup shows the presence of proteins reported for Ex in general and for Ex from B cells including CD20, Ig chains and MHC class II. We have previously reported that $\mathrm{ACTH}$, a melanocortin, and dextromethorphan (DM) and ANAVEX 2-73 (provided by ANAVEXTM Life Sciences Corp. under the SIGMACEPTOR® program), both sigma-1 receptor $(\sigma-1 \mathrm{R})$ agonists and weak NMDAR antagonists, protect OL, OL precursors (OPC) and neurons from death induced in vitro by excitatory (glutamate), apoptotic (staurosporine), reactive oxygen species (ROS) and quinolinic acid (a product of tryptophan indoleamine metabolism associated with inflammation). In preliminary studies we find that incubation of OL cultures with ACTH, DM and Anavex2-73 inhibits cell death induced by MS B cell Sup. Identification of the toxic factor/s and how they are released might provide a novel and more specific approach to B cell therapy in MS, and the OL and neuronal cytotoxicity assays might be useful in identification of molecules capable of inhibiting cortical gray matter damage in MS.

Title: Surviving Cell Death: The Difference Between Tolerance and Autoimmunity.

Authors: Linda Kusner, Henry J Kaminski - George Washington University; Robert Fenstermaker, Michael Ciesielski - Roswell Park Cancer Institute and Mmivax, Inc.

Abstract: Myasthenia gravis is a T cell/B cell-mediated disease. Therapeutics to reduce the autoantibody immune cells have focused on a general population of cells. We hypothesize that a target to an autoantibody-producing B cell will eliminate the population and lower antibody levels. Survivin is a 16.5$\mathrm{kDa}$ intracellular protein that belongs to the inhibitor of apoptosis protein family. We have evidence that patients with autoimmune MG possess survivin-positive B cells and hypothesized that therapy focused to eliminate these cells could be therapeutic. We pretreated Lewis female rats with survivin peptide mimic in incomplete Freunds' adjuvant before being induced with active EAMG. Rats were assessed biweekly for strength and weight. Pre-treatment with SVN53-67/M57$\mathrm{KLH}$ prevented weight loss in rats. Blinded evaluation found statistically significantly ( $p<0.05, t$ test) reduced weakness with average clinical scores of $0.25 \pm .46$ in the treated group and $1.3 \pm 1.0$ in the PBS control group. Animals were sacrificed 47 days after AChR injection. Analysis of the membrane attack complex (MAC) deposition at the NMJs from diaphragms of the pre-treated SVN53-67/M57-KLH (19.15 \pm 7.70 -pixel density) and PBS control (22.40 \pm 7.02 -pixel density) showed statistically significant ( $p<0.001$, MannWhitney $U$ ) reduction by density scan analysis performed alongside bungarotoxin to mark NMJs. ELISAs were performed to determine AChR specific total $\operatorname{IgG}, \operatorname{IgG} 1$ and IgG2b. The EAMG SVN53-67/M57-KLH treated animals 
demonstrated statistically ( $p<0.02, t$ test) lower average amounts of AChR antibody $(0.46 \pm 0.13,450 \mathrm{~nm})$ compared to the average amount of AChR antibody in control EAMG PBS treated animals $(0.68 \pm 0.13,450 \mathrm{~nm})$. The AChR specific IgG1and IgG2b were also statistically lower $(p<0.02, t$ test) compared to PBS controls. The results demonstrate that survivin peptide mimic could be a therapeutic for myasthenia gravis.

Title: Advancing Targeted Therapies for Patient Populations Harboring Ultra-Rare Mutations in a Rare Disease: Sarepta's Exon-Skipping Platform for Duchenne Muscular Dystrophy

Authors: Guriqbal S. Basi, Diane Berry, Mike Carver, Jay Charleston, Diane Frank, Doug Sanders, Fred Schnell, Saleh El Husayni, Joe Rutkowski, Jianbo Zhang

Sarepta Therapeutics, Inc. Cambridge, MA.

Abstract: Duchenne muscular dystrophy (DMD) is a rare, fatal, X-linked, degenerative neuromuscular disorder that affects 1 in every 3500-5000 male children worldwide. It is caused by mutations in the $D M D$ gene encoding dystrophin. Over 7000 different mutations have been reported in DMD patients manifesting as muscular weakness, followed by progressive muscle loss, and culminating in premature death attributable to respiratory or cardiac failure. The vast majority of mutations are exon deletions, resulting in out-of-frame mRNA, or nonsense mutations, all resulting in nonfunctional dystrophin. Antisense oligonucleotide-mediated exon skipping has been a viable strategy for restoring the reading frame by effecting splicing of the dystrophin pre-mRNA. Proof of concept studies in pre-clinical models supported clinical development of exon skipping oligonucleotides as therapeutic treatments for DMD. Exondys 51® (eteplirsen) is commercially available in the USA for treatment of DMD patients with a mutation in dystrophin amenable to exon 51 skipping. Exon skipping is a mutation-specific approach that potentially could address up to $80 \%$ of DMD patients. Consequently, each oligonucleotide applies to a subgroup of patients. Due to differences in frequency, mutations in some exons are more prevalent in some DMD patients than others, e.g., exon 51 amenable patients represent $13 \%$ of all DMD patients. Additional oligonucleotides targeting rare mutations, each accounting for $<1 \%$ of DMD patients, are applicable to decreasing patient numbers. Clinical development becomes increasingly more challenging as patient numbers decrease as traditional randomized clinical trials may not be feasible in DMD subpopulations with limited patient numbers. To address this challenge and provide therapeutic options to all DMD patients harboring mutations amenable to exon skipping, Sarepta is committed to building a robust knowledge base demonstrating safety, tolerability, and efficacy of phosphorodiamidate morpholino oligonucleotides (PMOs) as a therapeutic class.
Building on the foundation established by eteplirsen, preclinical and clinical understanding of safety, tolerability and efficacy of PMOs continues with golodirsen and casimersen, which treat patients amenable to skipping of exon $53(8 \%$ of all DMD patients) or 45 (8\% of all DMD patients), respectively. Even though eteplirsen, golodirsen, and casimersen are structurally unique, these three antisense oligonucleotidesall PMOs-exhibit similar pharmacokinetic and safety/ tolerability properties in pre-clinical studies. No adverse events attributable to the study drug were reported in phase 1 clinical trials of these PMOs. It is envisioned that the growing body of evidence from these three PMOs collectively targeting $29 \%$ of the patient population could be leveraged to facilitate development and approval of PMOs targeting mutations representing smaller patient sizes. This represents an innovative yet reasoned process to approval that mitigates unwarranted delay in providing patients access to life-saving or life-prolonging therapies, for which the risk vs. benefit equation regarding the drug target, as well as the therapeutic class, in a defined patient population, is well understood.

Title: RNA Trans-Splicing: Gene Editing for the Brain Author: Lloyd Mitchell, MD RetroTherapy

Abstract: RetroTherapy's unique RNA editing technology overcomes major problems associated with DNA editing: (1) Size RNA trans-splicing does not require delivery of any enzyme, such as Cas, which is large and potentially antigenic; (2) Cell division is not required for trans-splicing, a major advantage for any potential neurotherapeutic; and (3) Offtarget trans-spliced mRNAs are transient whereas off-target DNA editing is permanent. RetroTherapy specializes in the design and optimization of proprietary RNA trans-splicing molecules (RTMs) that invade the splicing of a chosen premessenger RNA (pre-mRNA) target, repairing or completely changing the coding sequence of the resulting mRNA. RTMs typically are delivered as DNA and have been expressed by most clinically relevant gene delivery systems, including AAV and lentiviruses for long-term therapy. One RTM can replace $>30$ exons, reprogramming many thousands of nucleotides. Therefore, 1-3 gene-specific RTMs could repair virtually all mutations within any gene and produce full-length repaired protein. All forms of gene editing provide key advantages over traditional gene therapy; (1) Size - only a portion of the gene to be repaired is required to be delivered;2) Expression - the repaired/replaced gene retains the natural regulation of the target gene; thus, it is expressed in the right place, amount and time; (3) Dominant negative mutations can be corrected. To date, 26 disease-associated genes have been trans-spliced including tau (frontotemporal dementia and Alzheimer's disease), rhodopsin and CEP-290 (blindness), SMN (spinal motor atrophy), and HTT (Huntington's) in 
relevant animal models. Trans-splicing has also been used to reprogram genes that cause cancer (p53, CD22, HPV, hCG), AIDS (HIV), cystic fibrosis (CFTR), progeria (lamin A), muscular dystrophy (dystrophin, plectin, titan, dysferlin, DMPK), hemophilia (factor 8), and severe combined immunodeficiency (CD40L, DNA-PK). RetroTherapy utilizes highthroughput screens to identify the most efficient and specific RTMs from custom-built libraries containing over a million gene specific RTMs. We are optimizing RTMs to repair HTT, LRRK2 (Parkinson's) and the dopamine D2 receptor. RetroTherapy has received funding from NIH, TEDCO, and MD Stem Cell Fund and is seeking investors and collaborative partners to accelerate the development of our platform technology.

Title: Lipid-shelled microbubbles for ultrasound-triggered release of Xenon to treat stroke

Authors: Christy K. Holland ${ }^{1,2}$ Arunkumar Palaniappan ${ }^{1}$ Himanshu Shekhar ${ }^{1}$ Department of Internal Medicine, Division of Cardiovascular Health and Disease, University of Cincinnati, Cincinnati, OH, USA; ${ }^{2}$ Department of Biomedical Engineering, University of Cincinnati, Cincinnati, OH, USA.

\begin{abstract}
Ischemia-reperfusion-induced neurological injury is a primary cause of stroke disability. Xenon (Xe), a bioactive gas, has potential as an effective and nontoxic neuroprotectant for the treatment of ischemic stroke [https://doi.org/10.1161/ CIRCULATIONAHA.109.879338]. When Xe is delivered by inhalation, high concentrations $(50-70 \% \mathrm{v} / \mathrm{v})$ are necessary to obtain a demonstrable neurologic effect, but limit the fraction of inspired oxygen. The goal of this work was to develop Xeloaded lipid-shelled microbubbles (XeMB) for site-specific release of Xenon upon pulsed ultrasound exposure in the neurovasculature. By encapsulating Xe into micron-sized lipid-shelled microbubbles, the therapeutic gas can be shuttled through the vasculature until delivery is triggered by ultrasound exposure. XeMBs were synthesized by high-shear mixing of $1 \mathrm{ml}$ lipid dispersion (9:1 $\mathrm{M}$ ratio of DSPC and 18:0 PEG2000 PE) in a vial that contained $1 \mathrm{ml} \mathrm{Xe}$, or a combination of Xe and octofluoropropane (OFP) (90/10\% v/ $v)$, in the headspace. The size distribution and acoustic attenuation spectrum of XeMB were measured using a Coulter counter and broadband attenuation spectroscopy (over 2$25 \mathrm{MHz}$ ), respectively. In vitro attenuation spectroscopy measurements demonstrate the feasibility of $6-\mathrm{MHz}$ pulsed Doppler ultrasound-triggered release of Xe from XeMB. The addition of octofluoropropane in the lipid-shelled microbubbles increased the number density, size, and stability of the microbubbles, particularly in undersaturated saline $(\sim$ $90 \pm 2 \%$ pO2), typical of the venous system. Gas chromatography-mass spectrometry was employed to
\end{abstract}

measure Xe dose $(120 \pm 5 \mu \mathrm{Xe} / \mathrm{mg}$ lipid). Intravenous administration of microbubbles carrying a neuroprotective gas in combination with ultrasound exposure has potential as a novel noninvasive strategy for local therapeutic delivery to modulate the effects of cerebral ischemia.

Title: EG-1962 for the Treatment of Aneurysmal Subarachnoid Hemorrhage

Author: Daniel Brennan, Edge Therapeutics, Inc.

Abstract: Edge Therapeutics, Inc. (EDGE) is a clinical-stage biotechnology company that discovers, develops and seeks to commercialize novel, hospital-based therapies capable of transforming treatment paradigms for the management of acute, life-threatening neurological and other conditions. EG-1962, Edge's lead product candidate, has the potential to fundamentally improve patient outcomes and transform the management of aneurysmal subarachnoid hemorrhage (aSAH), which is bleeding around the brain due to a ruptured brain aneurysm. In the USA, approximately 35,000 patients with an average age of 52 experience a subarachnoid hemorrhage (SAH) each year. Of these, approximately 20,000 experience an aSAH and are hospitalized in the intensive care unit, or ICU. As part of their routine course of treatment, up to half of aSAH patients have an external ventricular drain (EVD) inserted into the brain to monitor and relieve intracranial pressure by draining out cerebral spinal fluid. After the ruptured aneurysm is repaired, these patients remain at risk for multiple complications that are managed over the course of the patient's treatment to optimize clinical outcomes. Approximately $75 \%$ of aSAH patients suffer permanent brain damage or die within 30 days. An analysis of the 2013 National Inpatient Sample Database showed that the aSAH patients who require an EVD, and undergo either neurosurgical micro clipping or endovascular coiling for repair of their aneurysm, had the highest per-patient hospital charges, exceeding $\$ 400,000$ per patient, and experienced the longest hospitalizations of all aSAH patients. Not included in this analysis are the additional costs of rehabilitation, the longterm lifetime cost of illness related to an aSAH, the frequent inability to re-enter the workforce, or the potential need for additional care at home from family members or long-term caregivers associated with chronically disabled patients, which present additional significant economic burden to the healthcare system and the families of those afflicted with aSAH. Oral nimodipine is standard of care therapy to reduce the risk of poor outcome and secondary ischemia after aSAH. EG-1962 delivers high concentrations of nimodipine in a single dose directly to the brain administered via the EVD in a formulation that provides sustained drug exposure over 21 days. EG-1962 utilizes our proprietary, programmable, biodegradable polymer-based Precisa ${ }^{\mathrm{TM}}$ platform, through a 
novel delivery mechanism that enables targeted and sustained drug exposure while potentially avoiding dose-limiting side effects associated with currently available formulations of nimodipine. EG-1962 has been granted orphan drug designation and Fast Track designation by the U.S. Food and Drug Administration, or FDA, for the treatment of patients with SAH. The NEWTON study was a multicenter, randomized, controlled, open-label phase $1 / 2$ study, that evaluated the safety, tolerability and pharmacokinetics of escalating doses of EG-1962 compared to the current standard of care, oral nimodipine, in subjects with an aSAH. Clinical outcome results of the NEWTON study showed that $59 \%$ of patients treated with EG-1962 achieved a favorable outcome (scores of 6-8 as measured by the Extended Glasgow Outcome Score [GOSE]) at 90 days, compared to $28 \%$ of patients in the active control standard of care, oral nimodipine, arm who achieved a favorable outcome. In addition, improved clinical outcome was supported by a reduction in vasospasm, delayed cerebral ischemia, reduced use of rescue therapies, shorter intensive care unit (ICU) and overall hospital lengths of stay. Safety results showed that no patients experienced EG-1962 related hypotension, compared to $17 \%$ of patients treated with oral nimodipine. The ongoing pivotal, phase 3 NEWTON 2 study compares the efficacy and safety of EG-1962 to standard of care oral nimodipine in up to approximately 374 adult patients with aSAH.

Title: Discovery and Development of NPT520-34, A Novel Therapeutic Candidate for the Treatment of Parkinson's Disease and Related Neurodegenerative Disorders

Authors: Diana Price, PhD; Neuropore Therapies, Inc.; Asma Khan, Wolfgang Wrasidlo, Douglas Bonhaus Neuropore Therapies, Inc.

\begin{abstract}
NPT520-34 is a novel small molecule that was selected from a cell-based phenotypic screen designed to identify compounds that enhanced alpha-synuclein (ASYN) clearance. Further evaluation has determined that NPT520-34 is orally bioavailable and brain-penetrating compound with favorable ADME and safety profiles. One and 3 month in vivo efficacy studies utilizing the mThy1ASYN transgenic mouse model of Parkinson's disease (PD) were conducted to evaluate the effects of NPT520-34 administration on ASYN neuropathology, motor performance and potentially translatable CNS imaging markers including translocator protein (TSPO) as a marker of inflammation, and striatal dopamine transporters (DAT). NPT520-34 administration at 5 and $10 \mathrm{mg} / \mathrm{kg}$ ( 1 or 3 months) produced statistically significant reductions in the accumulation of aggregated ASYN, reductions in TSPO, and improved striatal DAT levels in transgenic mice. In addition, reductions in ASYN in the 3-month study were accompanied by
\end{abstract}

statistically significant improvements in grip strength in mice treated with 5 or $10 \mathrm{mg} / \mathrm{kg}$ NPT520-34. In conclusion, NPT520-34 treatment in an animal model of PD reduces ASYN neuropathology, produces functional motor benefit, and improves clinically tractable imaging biomarkers that will be used to guide clinical dose selection and serve as clinical markers of target engagement and efficacy. Currently, NPT520-34 is in preclinical development with an IND filing for NPT520-34 projected for 1Q19.

Title: Glial Cell Line-Derived Neurotrophic Factor (GDNF) in Parkinson's: Emerging Evidence of Clinical Benefit

Authors: Erich Mohr, PhD, R. Psych, MedGenesis Therapeutix Inc.; GDNF Study Group: Alan Whone, Matthias Luz, Mihaela Boca, Max Woolley, Lucy Mooney, Sonali Dharia, Jack Broadfoot, David Cronin, Christian Schroers, Neil U. Barua, Lara Longpre, C. Lynn Barclay, Chris Boiko, Greg A. Johnson, H. Christian Fibiger, Rob Harrison, Owen Lewis, Gemma Pritchard, Mike Howell, Charlie Irving, David Johnson, Suk Kinch, Christopher Marshall, Andrew D. Lawrence, Stephan Blinder, Vesna Sossi, A. Jon Stoessl, Paul Skinner, Erich Mohr, and Steven S. Gill

Abstract: We performed two studies testing intermittent intraputamenal convection-enhanced delivery (CED) of GDNF in 41 subjects with idiopathic Parkinson's: a 40week placebo-controlled, randomized, double-blind study and a 40-week open-label, all-active extension. At week 40 , GDNF was not superior to placebo on the primary endpoint (mean percent change in OFF state UPDRS motor score: $20.5 \pm 18.6 \%$ vs. $12.7 \pm 15.2 \%, p=0.13$ ) and secondary endpoints, although 18F-DOPA uptake was substantially improved with GDNF but not placebo in all putaminal regions $(p<0.0001)$. Despite the negative outcome, clinical results were consistent across endpoints and a post hoc responder analysis showed significant benefit for GDNF with a large clinically important motor response $(\geq$ 10 points: 9 [43\%] vs. 0 subjects; $p=0.0008$ ). At week 80 , OFF state UPDRS scores were markedly improved over baseline in both groups (GDNF/GDNF and placebo/ GDNF; motor: 9.6 vs. 9.0 points; ADL: 6.9 vs. 4.6 points). Also, week 80 scores in the GDNF/GDNF group were significantly better than week 40 scores in the placebo group (motor: 9.6 vs. 3.8 points, $p=0.0108$; ADL: 6.9 vs. 1.0 points, $p=0.0003)$. Although interpretation is limited by the switch from placebo to GDNF in the control group, we suggest that our results are indicative of neurorestoration leading to delayed clinical benefit. Prospective corroboration of our findings in a multi-center, randomized, double-blind 80 -week study, including a higher dose, is planned. 
Title: Lisuride-A Drug with Outstanding Affinity for Monoamine Receptors

Authors: Reinhard Horowski, MD, PhD, Sinoxa Pharma $\mathrm{GmbH}$

\begin{abstract}
The alpha-amino-ergoline lisuride (LIS), an "ergot antagonist", has a half-life of $2 \mathrm{~h}$. with no active metabolites, but a very variable first-pass effect when given p.o. This makes LIS an excellent candidate for parenteral application forms (s.c. with a portable minipump or a patch-pump or applied as a patch), e.g. to achieve continuous dopaminergic stimulation in PD with a daily dose of only about $1 \mathrm{mg}$. With these application forms excellent results have been achieved in PD and RLS. LIS has a strong $\mathrm{DA}_{2}$ and $5-\mathrm{HT}_{1 \mathrm{~A}}$ receptor affinity acting as an agonist and thus in PD combines antiakinetic and anti-dyskinetic effects. With a pump, in a doubleblind double-dummy pivotal study vs. oral agonists, LIS indeed has shown simultaneously significant superiority in UPDRS II and III as well as in UPDRS IV. In RLS, patches as small as $10-20 \mathrm{~cm}^{2}$ showed significant superiority over ropinirole p.o. As a prolactin-lowering drug, LIS at dosages of $0.2 \mathrm{mg}$ p.o. and $0.05 \mathrm{mg}$ s.c. inhibits the activity of prolactinomas and causes them to shrink. It also improves several benign breast diseases, known risk factors for breast cancer. As an unsurmountable 5- $\mathrm{HT}_{2 \mathrm{~B}}$ antagonist at lower than $10^{8} \mathrm{M}$ concentrations, LIS never has caused any cardiac valvulopathies. On the contrary, LIS has potent anti-fibrotic effects in animal models of, e.g. systemic sclerosis. As a 5$\mathrm{HT}_{2 \mathrm{~A}}$ antagonist, LIS inhibits secondary platelet aggregation as well as migraine attacks (here at $3 \times 0.025 \mathrm{mg} /$ day p.o.). Whilst marketed in its oral form as an anti-PD drug, LIS in more than 400,000 patients' years of chronic use never has caused organ fibrosis, severe or irreversible adverse events or any other persisting organ damage. Clinical development is ongoing.
\end{abstract}

Title: The Clinical Development and Therapeutic Potential of AXS-05 for Neuropsychiatric Disorders

Authors: Cedric O'Gorman, Amanda Jones, Kellie Kennon, Herriot Tabuteau Axsome Therapeutics Inc.

\begin{abstract}
AXS-05, a novel, oral, fixed-dose combination of dextromethorphan and bupropion, is in late-stage clinical development for treatment-resistant depression and agitation associated with Alzheimer's disease. Dextromethorphan acts as an NMDA receptor antagonist, a sigma-1 receptor agonist and a reuptake inhibitor of both serotonin and norepinephrine. Dextromethorphan is extensively metabolized in humans. Plasma concentrations proposed for psychotherapeutic effects are only achieved in the presence of metabolic inhibition. Bupropion inhibits the breakdown of dextromethorphan in humans via CYP2D6. Bupropion is also an established
\end{abstract}

antidepressant which acts as a dopamine and norepinephrine reuptake inhibitor. Both dextromethorphan and bupropion are nicotinic acetylcholine receptor antagonists. Clinical evidence with several classes of antidepressants, which individually share the mechanisms of action of AXS-05, support its development for treatment-resistant depression. Furthermore, the NMDA receptor antagonist property of AXS-05 may hold the potential for rapid onset of action based on the clinical experience with the prototypical NMDA receptor antagonist ketamine. Clinical evidence also suggests that glutamate transmission may play a role in the behavioral and cognitive changes in dementia. Clinical data with agents that, like AXS05, target sigma-1 (e.g. fluvoxamine, donepezil) have shown effects in patients with behavioral disorders and Alzheimer's disease (AD). The role for the serotonergic properties of AXS05 in AD patients with agitation is supported by clinical trial results with citalopram in this indication. Dextromethorphan has previously been reported, in the presence of metabolic inhibition, to reduce depressive symptoms in patients with TRD, AD and pseudobulbar affect, and to reduce agitation symptoms in patients with AD. Several phase 1 trials assessed the pharmacokinetics of AXS-05, dextromethorphan and bupropion, as well as the safety and tolerability of AXS-05. A phase 3 randomized, double-blind active-controlled 12week study is evaluating AXS-05 for the treatment of TRD. The primary efficacy outcome measure is the MontgomeryÂsberg Depression Rating Scale (MADRS). Additionally, a phase $2 / 3$, randomized, double-blind, placebo and activecontrolled (bupropion) 5 -week study is evaluating AXS-05 for the treatment of agitation associated with Alzheimer's disease. The primary efficacy outcome measure is the CohenMansfield Agitation Inventory (CMAI). Administration of AXS-05 resulted in substantial increases in dextromethorphan plasma concentrations in phase 1 trials. AXS-05 was safe and generally well tolerated. The STRIDE-1 study in patients with TRD is expected to enroll approximately 350 subjects. The ADVANCE-1 study in patients with AD is expected to enroll approximately 435 subjects. AXS-05 represents a novel innovative approach to the treatment of a number of neuropsychiatric disorders given the potential therapeutic synergy of the many clinically relevant mechanisms of actions of dextromethorphan and bupropion.

Title: Identifying Early Clinical Development Priorities for Disease-Modifying Therapies in Neurodegenerative Diseases Authors: Ludy Shih, MD, Biogen, Inc.; Miroslaw Brys; Laura Fanning; Tara Fox; Beth Hirschhorn; Shavy Makh; Minhua Yang; James Xiao; Karl Evans; Natasha Penner; Danielle Graham; George Nomikos; Jesse Cedarbaum.

Abstract: A common challenge for the clinical development of potential disease-modifying therapies for 
neurodegenerative diseases like Parkinson's disease (PD) is the need to demonstrate a clinically significant impact on the disease typically in its earliest stages when chances of therapeutic success are highest. Demonstration of target engagement and proof-of-biology are critical factors in earliest stages of development but are not sufficient. We carried out a doubleblind, placebo-controlled, single ascending-dose study of the anti-alpha-synuclein monoclonal antibody BIIB054 administered intravenously as a single dose at 1, 5, 15, 4, 90 and $135 \mathrm{mg} / \mathrm{kg}$ in healthy volunteers to evaluate its safety, pharmacokinetics and immunogenicity. Seven (50\%) of the 14 subjects receiving placebo and $19(56 \%)$ of the 34 subjects receiving BIIB054 reported at least one adverse event (AE). Most reported AEs were mild (CTCAE grade 1). One subject in the $135 \mathrm{mg} / \mathrm{kg}$ cohort had a serious adverse event (CTCAE grade 3 ). There were no clinically significant treatmentemergent abnormalities in vital signs, laboratory results or ECGs. The results of this study demonstrated that BIIB054 was safe and well tolerated in healthy volunteers in doses up to and including $90 \mathrm{mg} / \mathrm{kg}$ and provided rationale for further development. Based on these results and re-analysis of data from the Parkinson's Progression Markers Initiative (PPMI) study, we outline several approaches to further development of BIIB054, including designing and conducting a multicenter, randomized, double-blind, placebo-controlled study, with an active-treatment dose-blinded period to evaluate the longterm safety, pharmacokinetics, and pharmacodynamics of BIIB054 in subjects with PD.

Title: 2DG Update: Anticonvulsant Action, Neuroprotection, and Prevention of Post-Traumatic Epilepsy

Author: Thomas Sutula, MD, PhD, University of Wisconsin, Neurogenomex, Inc., Madison, WI

Abstract: 2DG (2-deoxy-D-glucose) is a glucose analogue
and reversible inhibitor of glycolysis currently in preclinical
development for therapeutic indications including epilepsy
and TBI. 2DG undergoes activity-dependent uptake into focal
brain regions in response to increased energy needs in neural
circuits during seizures and in response to brain injury. The
acute anticonvulsant efficacy of 2DG has been demonstrated
in multiple in vivo and in vitro seizure models and indicates
broad anticonvulsant action against network synchronization.
2DG also has chronic antiepileptic 'disease-modifying' ac-
tions consisting of 2 -fold slowing of kindling progression in
rats observed with administration of 2DG as long as 15 min
after repeated evoked seizures due to enhanced postictal de-
livery by neurovascular coupling in neural circuits with high
energy needs. After TBI by controlled cortical impact (CCI) in
rats, 2DG reduces secondary damage progression measured
by MRI/DTI, prevents frequent post-traumatic focal and
generalized seizures in $~ 50 \%$ of kindling-susceptible rats, favorably reduces fear conditioning at 1 month after injury, and at 6 months reduces fear memory deficits and preserves spatial context fear memory formation. The mechanisms underlying these novel acute and chronic therapeutic actions against seizures, epilepsy progression, and consequences of TBI are distinctive compared to currently marketed anticonvulsants. The acute anticonvulsant mechanisms of 2DG include activity-dependent reduction in synaptic currents by a presynaptic mechanism. The mechanisms underlying the chronic actions of 2DG involve glycolytic metabolic regulation of seizure-induced and injuryinduced gene expression by the transcriptional repressor neuron-restrictive silencing factor (NRSF) and its redox sensor carboxy-terminal-binding protein (CtBP). Preclinical toxicological studies of 2DG in rats have confirmed dose-dependent, reversible cardiac myocyte vacuolation with features of reversible autophagy at high doses, which were not observed in dogs and appear to be speciesspecific. These toxicological observations and human oncology studies demonstrating safety and tolerability of $40 \mathrm{mg} / \mathrm{kg} /$ day doses for 14 days and longer support potential use of 2DG for therapeutic indications including status epilepticus, seizure clusters, combination with device therapy, and as a neuroprotectant to prevent or reduce delayed adverse consequences after TBI including post-traumatic epilepsy, PTSD, and consequences of concussion.

Title: Ganaxolone, an Investigational Neurosteroid Treatment for Children with CDKL5 Deficiency Disorder: Results from a Phase 2 Trial.

Authors: Michael Rogawski, MD, PhD, University of California, Davis; Lorianne Masuoka, MD, Marinus Pharmaceutical; Nicola Specchio, Michael Chez, Daniel Tarquinio, Jaakko Lappalainen, Julia Tsai

\begin{abstract}
:
The X-linked CDKL5 gene mutations are the cause of an early-onset epileptic encephalopathy known as CDKL5 deficiency disorder. Individuals with this disorder have difficult-to-control seizures. An open-label study is being conducted to explore safety and efficacy of ganaxolone in children with CDKL5 deficiency disorder associated epilepsy. NCT02358538 is an open-label phase 2 study, and 7 subjects with genetically confirmed CDKL5 deficiency disorder were enrolled at one Italian and three US centers. Following screening and baseline visits, the patients enrolled into a 26-week study during which the investigators dosed ganaxolone up to a target of $1800 \mathrm{mg} / \mathrm{day}$ (>30 kg body weight) or $63 \mathrm{mg} / \mathrm{kg} /$ day ( $<30 \mathrm{~kg}$ body weight). Following the 26-week study period, eligible patients are eligible to enroll into a 52-week extension. The primary efficacy measure is $\%$ change from baseline in the 28-day seizure frequency (total seizure count).
\end{abstract}


Preliminary Results as of January 15, 2018 indicate the median change in 28-day seizure frequency from baseline in the ITT (intent-to-treat) population (primary endpoint) was a decrease of $43 \%(n=7)$. The median change from baseline in seizure-free days in the ITT population (key secondary endpoint) was an increase of $78 \%(n=5$; two subjects cannot be calculated due to 0 baseline seizure-free days, but both had an increase). Four children met the criteria to enter the one-year study extension (completed 26-weeks of treatment with excellent seizure control). The Clinical Global Impression Scale rated by Investigators (CGI-I) and Caregivers (CGI-P) were consistent with seizure control for all the children. Ganaxolone was generally safe and well-tolerated with no serious adverse events. Two children discontinued prior to completing the 26-week treatment due to lack of efficacy. These preliminary results suggest that oral ganaxolone is safe and well-tolerated, and based on these preliminary efficacy data, a phase 3 study will be initiated in 2018 in children with CDKL5 deficiency disorder. A phase 2 study of IV ganaxolone is also ongoing in refractory status epileptics.

Title: BPN14770 Clinical Development for Fragile X Syndrome.

Authors: Mark Gurney, PhD, Tetra Discovery Partners Inc.; Michael Tranfaglia MD, FRAXA Research Foundation; Newbury Port, MA; Patrica Cogram PhD, IEB, Faculty of Science, University of Chile, Chile; Scott Reines MD PhD, Tetra Discovery Partners, Grand Rapids MI; Elizabeth BerryKravis MD PhD Rush University Medical Center, Chicago, IL

\begin{abstract}
BPN14770 is a first in class, phosphodiesterase-4D (PDE4D) subtype-selective allosteric inhibitor being developed for the treatment of Fragile X Syndrome (FXS). FXS patients display a range of neuropsychiatric symptoms including intellectual disability, delayed language acquisition, poor social interaction, hyperarousal, hypersensitivity, repetitive behaviors, disrupted sleep, attention deficit hyperactivity disorder and autism. Dysregulation of cAMP signaling occurs in multiple tissues. These behavioral and biochemical changes are modeled in adult male mice in which the fmrl gene has been deleted. When treated daily with BPN14770 fmr1 KO mice showed significantly increased brain cAMP, reduced hyperarousal in the open field, significantly increased frequency of social interaction, and significantly improved natural behaviors (nesting and marble burying). The benefit of BPN14770 persisted 2 weeks after washout of the drug suggesting the potential for disease modification. Chronic dosing with BPN14770 also improved spine morphology on layer 3 pyramidal cells in medial prefrontal cortex. In phase 1 clinical trials BPN14770 was well tolerated, displayed linear pharmacokinetics, and showed evidence of central activity. Based on these promising preclinical
\end{abstract}

and clinical data, an initial clinical study of BPN14770 is planned to enroll 30 adult male (18-45 years inclusive) FXS patients in a randomized, double-blind, placebo-controlled, two-period crossover study.

Title: CIMIT: A Unique Model for Accelerating Healthtech Innovations

Authors: Steve Schachter, MD, Consortia for Improving Medicine with Innovation \& Technology (CIMIT); John A. Parrish, CIMIT; Michael K. Dempsey, CIMIT; Diane Spiliotis, CIMIT; John Collins, CIMIT

Abstract: The Consortia for Improving Medicine with Innovation \& Technology (CIMIT; http://cimit.org/) was founded in 1998 by leading hospitals and universities in Boston as a "center-without-walls" to foster multidisociplinary collaborations that bridge silos of medicine and technology to improve patient care. CIMIT leverages technological expertise from academia, industry, and government agencies to target unmet medical needs through close collaborations among innovative clinicians, engineers, scientists, and implementation experts across institutions. CIMIT's first $\$ 50 \mathrm{M}$ of investments in projects resulted in (1) more than $\$ 500 \mathrm{M}$ in follow-on funding at CIMIT institutions plus another $\$ 600 \mathrm{M}$ in commercial investment; (2) over 460 issued US patents and 2300 publications; (3) over 70 NewCo's or commercial licenses with more than $20 \%$ receiving regulatory approval for human use. CIMIT created the CIMIT Innovation Guidance and Impact Tracking System (GAITS) in 2017 to help innovators navigate the complex journey of innovation in healthcare. GAITS adds significant guidance to teams by defining core sets of deliverables at each milestone in four domains critical to success in healthcare innovation: clinical, market/business, regulatory/ approvals, and technology. Based on its success, CIMIT has now grown to become a national consortium and portal for international groups to access and collaborate with the country's best MedTech communities.

Title: Is Neurological Disease Due to Dysfunctional Brain Oscillators?

Authors: Robert Black, PhD, Scion NeuroStim

Abstract: There is now general recognition that brain dynamics are underpinned by collective oscillatory states. It follows, therefore, that neurological disease can be modeled as dysfunctional brain oscillators. The test of any model is whether it has predictive power and, clinically, whether it informs delivery of therapy. The advent of vestibular sensory neuromodulation as a treatment for episodic migraine (EM) headache provides a specific example with which to examine 
this model. The vestibular system innervates, directly or indirectly, extensive brainstem, subcortical and cortical regions, including the sensory cortices via thalamic relays. Migraine pathophysiology is associated with hyperexcited sensory responses and some have hypothesized that aberrant sensory sensitivity precipitates the subsequent cascades resulting in headache. It is therefore reasonable to hypothesize that vestibular neuromodulation is interrupting or regularizing sensory hyperexcitability, but what is the mechanism of action (MOA)? Examining previous results using time-varying CVS therapy is supportive of a model whereby CVS entrains endogenous neuronal pathways, leading to neuroplastic modification over time. Black et al. demonstrated entrainment of a pontine pacing network using a CVS therapy device. Significantly, induced oscillations in cerebral blood flow velocity had a period matched to B waves, which show abnormal dynamics in migraineurs. Black et al. showed that the time-varying character of applied CVS was crucial for entrainment and that the effect was clearly vestibular in origin. Wilkinson et al. presented evidence of the efficacy of CVS therapy for EM as an adjuvant, suggesting that it acts through a parallel MOA. Recently, a study of Parkinson's disease patients, using the same CVS treatment paradigm as the EM study, provided strong evidence of durable gains in motor and non-motor assessments, strongly suggesting neuroplastic modification of the brain. Current neuromodulation approaches deliver stimulation via implanted electrodes, induced cortical currents, or conduction between large-area scalp electrodes. In these approaches, the applied stimulus is adjusted empirically, heedless of the actual underlying, fine-scale oscillatory dynamics. Vestibular neuromodulation is categorically different from current forms of neuromodulation in that it acts via a sensory system. Applied oscillatory modulation is carried and transformed via endogenous pathways so that the modulation effectively entrains distant oscillatory networks. A reasonable hypothesis, therefore, is that repeated entrainment with time-varying vestibular stimulation drives aberrant oscillatory networks back to ontogenetic baseline configurations through neuroplastic modification, reducing hyperexcitability in the case of migraine and, more generally, bringing network activity closer to pre-disease function. The extensive literature around the dynamics of collective oscillators will also be discussed in this context.

Title: Pharmacodynamic phMRI with Joint Estimation of the Arterial Drug Input Curve

Authors: Molly Charney, Washington University in St. Louis; Jonathan M. Koller; Brad Miller; Lauren A. Marks; Kevin J. Black

Abstract: This study introduces a refinement to a method for quantifying the sensitivity of multiple brain regions to a drug.
We previously described rapid quantitative pharmacodynamic imaging, which simultaneously estimates pharmacokinetic (PK) and pharmacodynamic (PD) parameters from a region of the brain. Here, we use the fact that the concentration of a drug in arterial blood is essentially the same for all regions of the brain to more tightly estimate the time-concentration curve and hence reduce unwanted model flexibility in estimating PD parameters. Furthermore, assuming linear pharmacokinetics, we can appropriately combine responses to the drug across doses. To test this approach, a dopamine D1-like agonist, SKF38393, was administered intravenously to macaques $(n=4)$ during BOLD-sensitive fMRI. Each subject received four different doses of drug on four different occasions. Data from the hypothalamus, midbrain, and striatum were used to estimate the most likely time-concentration curve; the $90 \%$ confidence interval for this curve was much tighter than the range of curves when data for each region and dose were considered one at a time (Figure). The most likely timeconcentration curve was then used as prior information to constrain quantification of drug sensitivity (EC50) at each point in brain. The results support the use of rapid quantitative pharmacological imaging in determining appropriate and effective dosing for brain disorders such as Parkinson's disease.

Title: Neuroprotective Methlthiazole DerivativesMechanisms and Comparative Efficacy

Authors: Doug Cowart, Pharm.D., Revivo Therapeutics, Inc.; Sue H. Lee, Manel BenAissa, Jia Luo, Gregory Thatcher

Abstract: Therapies are needed to treat neurodegenerative diseases such as Alzheimer's (AD), Parkinson's (PD) and traumatic brain injury (TBI). Nomethiazoles (NMZ) are NO analogues of the neuroprotective methiazole pharmacophore, with procognitive, anti-inflammatory and anxiolytic activity. NMZ are known to cross the blood-brain barrier and suppress activation of microglia and astrocytes, with direct procognitive activity. Anti-inflammatory and procognitive activity after chronic administration of NMZ was successfully validated in three mouse models of AD, including APP/PS1, $3 \times \mathrm{xTg}$, and a $5 \mathrm{xFAD}$ mouse expressing human APOE4. To test acute effects, in a novel model administered after mild traumatic brain injury (mTBI), NMZ acutely blocked both cytokine surge and cognitive loss. Sedation related to potentiation of chloride currents at GABAA and glycine receptors was dissociated from procognitive and anti-inflammatory activity in mouse models. The lead NMZ, RIV-5061, has been studied in humans, and second-generation NMZ replicate the actions of RIV-5061 in vitro and in vivo. The NO-initiated activity of NMZ is mediated by activation of cGMP signaling. Current preclinical studies to support the mechanism of action of NMZ are focused on comparison of NMZ with NO-independent drugs: (i) the parent methiazole and (ii) a cGMP activator. 
Ongoing preclinical studies will reveal the basis for the attenuation of hallmark $\mathrm{A}^{2}$ and tau pathology observed in all animal models studied to date with NMZ.

Title: Use of iPSC-derived Human Motor Neurons in HighThroughput Phenotypic Screening

Authors: Shouming Du, BD, BrainXell, INC; Michael Hendrickson, Zhong-Wei Du BrainXell, Inc., Madison, WI, Jennifer Kouznetsova, Wei Zheng NCATS/NIH, Rockville, MD

\begin{abstract}
There exists a dire need for more effective drugs to treat disorders of the central nervous system (CNS), and new cell culture models that are more relevant to psychiatric and neurological diseases are needed to improve the success rate of drug development programs. One such approach is the use of neurons differentiated from patient-induced pluripotent stem cells (iPSCs), which present new opportunities for modeling disease processes and screening drug libraries. We have developed technology to produce very large quantities of highly enriched human neurons from patient iPSCs and to greatly accelerate their maturation. We subsequently developed two high-throughput screening (HTS) systems using human motor neurons derived from individuals with amyotrophic lateral sclerosis (ALS) and spinal muscular atrophy (SMA). Using genome editing techniques on the iPSC lines, the reporter nanoluciferase (Nluc) was fused to endogenous neurofilament light chain (NFL) for ALS and full-length SMN2 for SMA. Nanoluciferase is more sensitive than the traditional luciferase and allows for a simple and quantitative readout of NFL and SMN2 protein levels. The assays were adapted to meet HTS requirements, including large batch sizes, 1536-well format, minimal well-to-well variation, short-term culture, plating by automated dispenser, and low reagent volumes. Applying a quantitative HTS approach, we screened the LOPAC, NPC, and MIPE libraries (> $6000 \mathrm{com}-$ pounds) in a dose-dependent manner on both motor neuron lines with a hit rate of $\sim 0.5 \%$. This work demonstrates the feasibility of running HTS campaigns using human neurons, which present a more physiologically relevant drug discovery platform.
\end{abstract}

Title: How the Epilepsy Foundation Supports Research Authors: Sonya Dumanis, Brandy E. Fureman, PhD, The Epilepsy Foundation

\begin{abstract}
The Epilepsy Foundation's research purpose is to develop an ecosystem that continually drives innovation forward to solve the challenges and remove the roadblocks that stand in the way of ending epilepsy. Here, we describe how the Foundation achieves this goal by investing in innovation,
\end{abstract}

engagement and next-generation scientist's programs. Our innovation programs support new ideas by taking on high-risk, high-reward projects through our innovation incubator known as the Epilepsy Innovation Institute. We also support a series of commercialization grants for later-stage development projects through our Epilepsy Therapy Project. These programs have supported the development of thermal ablation therapy (now acquired by Medtronic) and trigeminal nerve stimulation (now approved in the EU) for epilepsy. Our engagement programs improve communication between people with epilepsy, their families, advocates, researchers, regulators, and investors. We connect people with epilepsy to clinical trials via our Clinical Trial Portal. We created a learning network and data-bank through the Rare Epilepsy Network, which has over 1350 individuals enrolled. We work with regulators and industry to get therapies to the market faster with our annual Research Roundtable in Epilepsy. Since the 1960s, we have also supported the career development of epilepsy researchers. We have a proud partnership with the American Epilepsy Society, continuing this long-standing support.

Title: Pharmacokinetics of Clobazam Oral Soluble Film Authors: Allen H. Heller, Pharma Study Design LLC; Stephen Wargacki ${ }^{1}$, Cassie Jung ${ }^{1}$, David J. Wyatt, Syneos Health Clinical Solutions; A. Mark Schobel ${ }^{1},{ }^{1}$ Aquestive Therapeutics LLC

Abstract: Clobazam oral soluble film [COSF] is a novel dosage form under development for adjunctive treatment of seizures in Lennox-Gastaut syndrome. We assessed the pharmacokinetics (PK) and safety-tolerability of clobazam administered as single doses of COSF 20 and $10 \mathrm{mg}$ (Aquestive Therapeutics, LLC) compared with clobazam tablets 20 and $10 \mathrm{mg}$ (Lundbeck, USA). Healthy adult volunteers $(N=51)$ were enrolled in a single-dose, open-label, randomized foursequence, four-period, crossover: (A) COSF $20 \mathrm{mg}$; (B) Clobazam tablet $20 \mathrm{mg}$; (C) COSF $10 \mathrm{mg}$; and (D) Clobazam tablet $10 \mathrm{mg}$. PK sampling for clobazam and $\mathrm{N}$ desmethylclobazam was carried out until 21 days post-dose with a 28-day washout. Subjects were monitored for adverse events (AE) throughout the study. COSF at single doses of 10 and $20 \mathrm{mg}$ was bioequivalent to the clobazam tablet at equivalent doses for both clobazam and $N$-desmethylclobazam, its active metabolite. The PK of both formulations were doseproportional. AE were dose-related across the treatment groups, with somnolence the most common. Most events were mild and none were serious or severe. COSF is a novel clobazam dosage form that is bioequivalent to the clobazam tablet. Because of its ease of administration, COSF may be expected to improve adherence, reduce likelihood of error, and provide more accurate dosing than formulations of clobazam that are currently available. 
Title: Population Pharmacokinetic Modeling of Diazepam Buccal Soluble Film

Authors: Allen H. Heller, $\mathrm{MD}^{1}$; Stephen Wargacki ${ }^{2}$, Cassie Jung $^{2}$ : David J. Wyatt ${ }^{3}$ Guillaume Bonnefois ${ }^{3}$, Olivier Barrière $^{3}$, Pierre-Olivier Tremblay ${ }^{3}$, A. Mark Schobel ${ }^{2}$,

${ }^{1}$ Pharma Study Design, LLC; ${ }^{2}$ Aquestive Therapeutics LLC;

${ }^{3}$ Syneos Health Clinical Solutions

Abstract: Diazepam buccal soluble film (DBSF) is a novel dosage form of diazepam under development for the management of selected, refractory, patients with epilepsy, on stable regimens of AEDs, who require intermittent use of diazepam to control bouts of increased seizure activity. We have observed that diazepam pharmacokinetics (PK) following a single dose of DBSF are dose-proportional over the range of 5 to $15 \mathrm{mg}$ for both maximum plasma concentration (Cmax) and area under the curve (AUC). In contrast, diazepam PK following a single dose of diazepam rectal gel (DRG) are doseproportional for AUC, but less than dose-proportional for Cmax. To identify doses of DBSF expected to be therapeutically equivalent to labeled doses of DRG, a Population PK modeling (NONMEM 7.3) for DBSF and DRG was performed using PK data obtained in phase 1 studies in adult men and women. DBSF and DRG were separately modeled using the same structural model, a two-compartment model with Michaelis-Menten absorption, linear elimination, and mixed residual error. Using an algorithm intended to apply equal weight to matching on both Cmax and AUC (0-12 h), preliminary results indicate that DRG doses of 7.5. 10, 12.5, $15,17.5$, and $20 \mathrm{mg}$ are best matched by DBSF doses of 5, 7.5, 7.5, 10, 12.5, and $12.5 \mathrm{mg}$, respectively. This modeling approach provides the flexibility to match DBSF with DRG doses on any selected PK parameter(s).

Title: SCUBA: Subgroup Based Bayesian Adaptive Designs for Precision Clinical Trials

Author: Yuan Ji, NorthShore University Health System

Abstract: In precision medicine, a patient is treated with targeted therapies that are predicted to be effective based on the patient's baseline characteristics such as biomarker profiles. Oftentimes, patient subgroups are unknown and must be learned through inference using observed data. We present SCUBA, a Subgroup ClUster-based Bayesian Adaptive design aiming to fulfill two simultaneous goals in a clinical trial, (1) to treatments enrich the allocation of each subgroup of patients to their precision and desirable treatments and (2) to report multiple subgroup-treatment pairs (STPs). Using random partitions and semiparametric Bayesian models, SCUBA provides coherent and probabilistic assessment of potential patient subgroups and their associated targeted therapies. Each STP can then be used for future confirmatory studies for regulatory approval. Real-world examples in cancer and cardiovascular diseases will be presented. SCUBA can be applied in general to many different disease areas, as long as there is a need to match the patient biomarker profiles with specific treatment options.

Title: NNI-351, A First-in-Class Candidate for Diseasemodifying ADHD Treatment

Authors: Judith Kelleher-Andersson, Neuronascent, Inc., Matthew Wells, Novavax

Abstract: NNI-351, a novel small molecule agent was assessed for ADHD therapeutic benefit by determining cognitive and anti-hyperactive efficacy in a model of neurodevelopmental delay and through measurement of neurogenesis-related benefit in normal mice. NNI-351 was administered daily to partial-Trisomy mice (Ts65Dn) for a 4week period and changes in cognitive deficit, neurogenesis activity and hyperactivity were measured. Specifically, a Contextual-Object Recognition paradigm was used for cognitive changes, Ki67-labeled cells were counted in the dentate gyrus neuronal layer, and hyperactivity and stimulant-like activity measured using both open-field and plus-maze paradigms, all versus vehicle and wild-type controls. Normal mice, previously shown to promote in vivo hippocampal neurogenesis, were assessed for neuropsychiatric benefit. NNI-351 given either by oral or intraperitoneal administration showed cognitive benefit back to normal levels in Down syndrome-modeled mice and inhibited hyper-activity without stimulant-like activity versus wild-type levels. In normal mice, there was an improvement in cognition associated with previous neurogenic time frame. Efficacy by NNI-351, a firstin-class oral new chemical entity, in an ADHD-like model (Ts65Dn), might be beneficial for children and adults with ADHD over-marketed therapeutics.

Title: Therapeutic Exposures of CX-8998, a Potent, Selective and State-Dependent Cav3 Channel Antagonist with DoseDependent Efficacy in Cav3-Driven Neurological Models

Authors: Margaret Lee, Spyros Papapetropoulos, MD, PhD; Evan Newbold, PhD Cavion, Inc.

Abstract: In this study, we utilized preclinical data to establish predicted human therapeutic exposures of the potent, selective and state-dependent Cav3 channel antagonist, CX8998 , currently in phase 2 clinical studies for essential tremor and generalized epileptic syndrome with absence seizures. Cav3 channels are low-threshold voltage-gated calcium channels that regulate neuronal excitability and are genetically and pharmacologically validated targets for tremor, epilepsy and neuropathic pain. Cav3 is a mediator of subthreshold 
oscillations and excessive rhythmicity in pathophysiologic states found in tremor and is highly expressed in functional tremor network regions. Cav3 gain-of-function mutants are found in childhood absence epilepsy (AE) patients and genetic studies in mice have linked Cav3 channels to AE-like phenotypes. Rodent models of tremor and spontaneous absence epilepsy show enhanced Cav3 currents and burst-firing. Currently approved drugs, including zonisamide and ethosuximide, have been reported to have non-selective Cav3 antagonist activity and while both drugs have shown evidence of efficacy in clinical studies of tremor and epilepsy, many patients experience lack of efficacy or intolerable side effects resulting in treatment discontinuation. Thus, selective targeting of Cav3 channels has the potential to improve efficacy and tolerability in indications such as tremor and epilepsy where Cav3 is an important component of disease pathophysiology. We have investigated the potency and efficacy of CX-8998 and other Cav3 antagonists in rat preclinical models dependent on elevated Cav3 activity including harmalineinduced tremor and the WAG/Rij genetic model of spontaneous absence seizures. Dose- and time-dependent effects of Cav3 antagonists on tremor and seizure were objectively quantified using measures of tremor power and electrocorticogram recordings. Cav3 antagonists suppressed both tremor and accumulated time in seizure in a dose- and timedependent fashion. CX-8998 showed robust activity following single oral doses of $1-10 \mathrm{mg} / \mathrm{kg}$ with exposure-response analysis indicating an effective plasma concentration threshold of 150-350 nM CX-8998 in rat. Adjusting for species differences in plasma protein binding predicts a human therapeutic plasma concentration range of 300-700 nM. Comparison of Cav3 antagonist potencies suggests that CX8998 will result in substantially greater target engagement at achievable CNS concentrations compared to less potent and selective Cav3 antagonists zonisamide and ethosuximide. In conclusion, Cav3 antagonists, including CX-8998, show robust efficacy in preclinical models dependent on Cav3 pathology including tremor and epilepsy. Evaluation of preclinical efficacy and pharmacology data for CX-8998 supports an achievable effective human plasma concentration range and suggests the potential for improved Cav3 target engagement compared to less potent and selective antagonists.

Title: The Key Roles of Nigella Sativa-Oil in Regulating Microglia and Mitochondrial Activity in Parkinsonism

Authors: Tafheem Malik, MD, National University of Health Sciences; Tasneem Fatima, Sheem Hasan, Shahid Pervez, Darakhshan, Jabeen Haleem

Abstract: Aging increases the risk for a variety of neuronal alterations. Neuronal alterations increase in oxidative stress has been associated with the pathogenesis of Parkinsonism.
Oxidative stress induces pro-inflammation via increase resident immune responses such as microglial astrocytes. Microglia-mediated inflammatory responses and altered defensive action have been reported before any programmed neuronal death. Programmed death ensured if latency to alter defensive mechanism and precipitate into formation of disease, microglial support synaptic plasticity and maintain homeostasis. An ability to mitotically divide, self-renewal, and a telomere shortening demonstrate their capability to become senescent. An imbalance between antioxidant defense mechanisms and free radical production that overt the activity of innate resident immune cells occurs as age progresses and intensive in Parkinsonism. Nigella sativa-oil (NS) (black cumin seeds) and its constituentsa traditional medicine used for the seizure treatment, and in eastern countries - that effectively control neuronal inflammation and microglial proliferation and manage defensive control of astrocytes and has been well documented to exhibit antioxidant defense mechanisms. In this study, we investigate whether NS reduce the haloperidol (HAL)-induced microglial activation and Parkinsonism. After a combined treatment with HAL $(1 \mathrm{mg} / \mathrm{kg})$ on NS $(0.2 \mathrm{ml} / \mathrm{rat})$, rats displayed a significant reduced Parkinsonism and oral dyskinesia as compared to controls. Pyknotic neurons were histologically identified in HAL-treated rats which were absent in NS/HAL-treated animals. Immunohistochemical analysis indicates that NS reduced astrogliosis in caudate and accumbens nuclei. Activation of microglia together with reactive changes in astroglial morphology combats movement deficits. The prospective lab concerns are to understand mechanics of NS that may reduce pro-inflammatory cytokines responses, latent therapeutic targets on microglial cells, and mitochondriamediated crosstalk of astrocytes and microglia, worth investigating to recognize the effective treatments for Parkinsonism and other age-dependent inflammatory diseases of the CNS.

Title: ANAVEX®2-73, a Sigma-1 Receptor Agonist, Taking Aim at Precision Medicine to Treat Neurological Disorders

Authors: Emmanuel O Fadiran, Daniel Klamer; Nell Rebowe; Christopher Missling, Anavex Life Sciences Corporation

Abstract: After establishing a favorable safety profile of ANAVEX®2-73, a selective sigma-1 receptor agonist, drug concentration-response relation was observed in the phase $2 \mathrm{a}$ with the secondary exploratory endpoints MMSE and ADCS-ADL in mild-to-moderate Alzheimer's disease patients. These patients (MMSE baseline 16-28) were treated for 57 weeks. The same patients are continuing the same treatment regimen for additional 104 weeks (cumulated 
161 weeks) in the extension study. ANAVEX®2-73 demonstrated favorable safety and tolerability through cumulated 109 weeks, and Alzheimer's patients with highest drug concentrations had improved cognition and function during 57 weeks and retained response at cumulated 109 weeks. The objective is to continue to assess safety of ANAVEX ${ }^{\circledR 2-73}$ and to assess whether genetic biomarkers could be identified in order to pre-specify patients in future ANAVEX®2-73 studies under the precision medicine paradigm to assess if certain genes are linked to treatment response. Use of genetic data to enrich study populations has been extensively used in the development of oncology, however, not yet in the CNS. Hence, the full DNA and RNA of attainable Alzheimer's patients from the ongoing phase $2 \mathrm{a}$ extension study will be sequenced and analyzed. This precision medicine approach could be applied to future clinical studies of ANAVEX®2-73, like in Alzheimer's disease, Rett syndrome, and Parkinson's disease.

Title: A Phase I/II Trial of a Hepatocyte Growth Factor (HGF) DNA Plasmid for the Treatment of ALS

Authors: Marie-Laure Nevoret, VM BioPharma

\begin{abstract}
HGF is an angiogenic and neurotrophic multifunctional mesenchyme-derived cytokine. HGF and its cMET receptor are expressed in the peripheral nervous system, brain and spinal cord. VM202, a plasmid DNA expressing the two isoforms of HGF, may be beneficial in ALS.18 subjects with ALS (15 males, 3 females; mean age 52.4 years; average 12.8 months since diagnosis) were enrolled in a phase $1 / 2$, open-label clinical trial to assess safety and efficacy of VM202 intramuscular (IM) injections; 17 completed 9 months of follow-up. All 18 subjects tolerated $64 \mathrm{mg}$ VM202: Group1 $(n=9)$ received upper limb injections on Day0 and 14, and lower limb injections on Day7 and 21; Group2 received the alternate sequence. Preliminary efficacy was assessed via ALS Functional Rating Scale-Revised (ALSFRS-R), forced vital capacity (FVC), muscle strength, muscle circumference, and dynamometry. There were no adverse events (AE) or serious AEs related to study drug. Twelve subjects reported 26 mild or moderate injection site reactions. One subject died from respiratory insufficiency. Mean ALSFRS-R score at baseline was $38.7 \hat{\mathrm{A}} \pm 4.4$, and mean change in total ALSFRS-R score at 3 months for Group 1 was $0.65 \hat{\mathrm{A}} \pm 0.19$ points/month, a lower rate than literature reports. Responders (\% of patients whose ALSFRS-R improved or did not change from baseline) were 47, 50 and $24 \%$ at 1,2 and 3 months. FVC, muscle strength and dynamometry showed a plateau or slower decline from Day0 to Day90.VM202 appears safe and may stabilize ALS for 3 months.
\end{abstract}

Title: CX-8998: a Novel, State-Dependent Cav3 Channel Antagonist in Phase 2 Development for Tremor and Epilepsy Authors: Spyr Papapetropoulos, MD, PhD, Stacey Boyer, PhD Margaret Lee, PhD Cavion Inc.

Abstract: CX-8998 is an orally bioavailable, potent, statedependent and selective antagonist of T-type calcium channels (Cav3) currently in phase 2 development by Cavion Inc. Cav3 channels are preferentially expressed in CNS and PNS and activate upon small membrane depolarizations allowing a surge of calcium entry into excitable cells initiating the action potential and modulating neuronal firing. In pathologic states, Cav3 is either upregulated or found to have increased activity, making it a selective target for specific neurologic diseases such as tremor, epilepsy and pain. CX-8998 and its analogues have been extensively studied in in vitro and in vivo preclinical pharmacology models and have a robust preclinical toxicology and safety pharmacology package that support human clinical studies. Clinical development is also supported by a human safety database of more than 200 subjects. Two proof-of-concept (Ph2), randomized, double-blind, placebo-controlled, parallel-group studies of CX-8998 are currently ongoing in Essential Tremor (T-CALM), and Generalized Epileptic Syndromes with Absence Seizures (T-WAVE) are underway to evaluate titrating doses of CX-8998 up to $10 \mathrm{mg}$ BiD. These studies are expected to test the hypothesis whether selective and potent modulation of Cav3 with CX-8998, can improve symptom severity in ET patients and reduced the electrographic seizure burden in patients with generalized epilepsy. Readouts are expected in the second half of 2018.

Title: Network for Excellence in Neuroscience Clinical Trials, NeuroNEXT: Update on Success and 5-Year Renewal Application

Authors: Julie Qidwai, University of Iowa Clinical Trials Statistical and Data Management Center; Marianne Kearney Chase, Dixie Ecklund, Mike Bosch, Brenda Thornell, Christopher Coffey, Merit Cudkowicz

\begin{abstract}
The NINDS-funded NeuroNEXT (NN) Network consists of 25 clinical sites, a Data Coordinating Center (DCC) and Clinical Coordinating Center (CCC). NN is completing $\mathrm{Y} 7$ of its initial grant period and has partnered with industry, small business, and academia to initiate nine trials and present results from four clinical trials to date. The Network is under review for a 5 -year renewal. NN innovates across the clinical trial life cycle, from study design, to recruitment and retention, to study close-out. The network aids investigators in implementation of cutting-edge design and analysis techniques, including adaptive and
\end{abstract}


Bayesian designs and futility analyses. NN closely monitors efficiencies and provides sites feedback on key performance areas in the form of biannual report cards. The network as a whole has achieved an average time of 3.6 months from funding to database release; $96 \%$ data accuracy; $80 \%$ of data entered within 7 days; $80 \%$ of trial participants with no major protocol deviations; and across trials, the retention rate is $85 \%$. NN recruitment has consistently outpaced disease-specific recruitment norms. The network closed out three trials simultaneously in the second half of 2017, the result of deft collaboration between CCC, DCC and clinical site staff. New initiatives proposed in the renewal application include expanding investigator education opportunities and growing the $\mathrm{NN}$ by partnering with industry and disease foundations.

Title: Multi-modal Retention in a Phase III Clinical Trial in Early Parkinson's Disease (STEADY-PD III)

Authors: Tanya Simuni, MD, Northwestern University Feinberg School of Medicine; Robert Holloway, Karen Hodgeman, Brittany Greco, Susan Henderson, Jillian Lowell, Saloni Sharma, Christopher G. Tarolli, and Kevin Biglan on behalf of the STEADY-PD III Investigators of the Parkinson Study Group

\footnotetext{
Abstract:

To evaluate novel retention strategies in an ongoing phase 3 clinical trial in individuals with Parkinson disease (PD). Adequate participant retention is a significant barrier to the successful completion of phase 3 clinical trials. Length of study, uncertainty of treatment arm and efficacy, treatment side effects, and early participant fatigue contribute to poor retention and dropout leading to ambiguous results. STEADY-PD III is a three-year, multicenter, phase 3, placebo-controlled trial of isradipine as a disease-modifying agent in individuals with untreated PD. The study aimed to enroll 336 individuals, based on a power calculation accounting for up to $5 \%$ participant loss per year. Ongoing efforts to enhance retention include monthly investigator and coordinator conference calls to address retention; periodic study participant newsletters to inform participants of ongoing study activities and milestones; participant teleconferences to review study progress and address participant questions; participant appreciation gifts to encourage ongoing participation; and travel reimbursement for study participants. Three hundred thirty-six participants were enrolled in the study, and to date, $323(96 \%)$ participants have completed all study activities or remain active in the trial. All remaining active participants have completed at least 2 years of follow-up, and the final
}

participant is expected to complete the study in November 2018.

"In its ongoing phase."

Title: Th1 Cytokines, Secretion in Dementia: Correlation with Type and Severity of Disease

Authors: Benjamin Sredni, Bar Ilan University, KenigsbuchSredni D., Huberman M.

Abstract: The production of interleukin-2 (IL-2) and interleukin-6 (IL-6) by peripheral blood mononuclear cells (MNC) was assessed in patients with Alzheimer's disease (AD) who were subdivided into two groups - mild and moderately-severe - according to the severity of the disease, probable vascular dementia $(\mathrm{VaD})$ patients and elderly control subjects. No differences in IL-2 secretion were found between mild AD patients and controls. However, there was a significant increase in IL-2 and gamma interferon (IFN-gamma) secretion both in the moderately-severe $\mathrm{AD}$ group and in the VaD group. IL-6 levels in AD patients of both groups were similar and significantly higher than those of $\mathrm{VaD}$ and controls. Our results suggest that increased levels of Th1 cytokines, IL-2 and gamma interferon (IFN-gamma) production correlate with severity of the dementia, whereas increased levels of IL-6 production seem to be related to AD and thus may play a role in $\mathrm{AD}$ pathogenesis.

Title: A Phase IIA, Randomized, Double-Blind, PlaceboControlled, 3-ARM Parallel-Group, Multicenter Study with Active Immunotherapeutic Vaccine UB-311 in Patients with Mild Alzheimer's Disease

Authors: Ajay Verma ${ }^{1}$, H. J. Yu ${ }^{1}$, P. N. Wang, Taipei Veterans General Hospital; M. J. Chiu, National Taiwan University Hospital; C. C. Huang, Linkou Chang Gung Memorial Hospital; C. C. Chang, Kaohsiung Chang Gung Memorial Hospital; H.C. Chen ${ }^{1}$, C. Y. Wang ${ }^{1},{ }^{1}$ United Neuroscience Ltd.

Abstract: UB-311 is an active vaccine targeting the A $\hat{I}^{2} 1-14$ epitope currently in a phase IIa trial (V203-AD) fully enrolled with subjects with mild Alzheimer's disease (AD). The primary endpoints are safety, tolerability and immunogenicity of two different dosing regimens of UB-311 (initial three priming doses followed by either four booster doses given every 12 weeks or two booster doses given every 24 weeks) compared to a placebo group. Secondary outcomes include changes from baseline in cognitive, imaging, functional, and global assessments through the end of the study. In a completed phase 1 clinical study, UB-311 was well tolerated and safely elicited high anti-A $\hat{\mathrm{I}}^{2}$ antibody levels with a $100 \%$ responder rate in patients with 
mild-moderate $\mathrm{AD}(n=19)$. A total of 43 subjects enrolled in the ongoing phase IIa study. For the $31(72.1 \%)$ subjects who have completed the treatment period (week 60) in the ongoing phase IIa trial in amyloid PET scan-positive patients, no incidences of treatment-induced meningoencephalitis or brain edema (ARIA-E) were detected from 220 post-vaccination MRI reads. To date, UB-311 has been well tolerated, as continuously assessed by clinical exam and MRI, with over 200 vaccine doses administered. Subjects from the phase $2 \mathrm{a}$ study will be eligible to join a 108-week extension study with UB-311. Results from this phase IIa study are expected in Q4 2018.

Title: Intravenous Allopregnanolone for Treatment of Status Epilepticus: Safety and Pharmacokinetics. in Dogs

Authors: Irene Vuu, University of Minnesota College of Pharmacy; Coles LD ${ }^{1,2 ;}$ Leppik, IE ${ }^{2}$.

Rogawski, MA ${ }^{4 ;}$ Zolkowska, D ${ }^{4}$; Wu C-Y ${ }^{4}$; Patterson, $\mathrm{EE}^{3}$; Cloyd, JC ${ }^{1,2}{ }^{1}$ Center for Orphan Drug Research, University of Minnesota, Minneapolis, Minnesota, ${ }^{2}$ Department of Experimental and Clinical Pharmacology, College of Pharmacy, University of Minnesota, Minneapolis, Minnesota, ${ }^{3}$ College of Veterinary Medicine, University of Minnesota, Minneapolis, Minnesota, ${ }^{4}$ University of California at Davis, Department of Neurology, Sacramento, CA

Abstract: Allopregnanolone (ALLO) is a neurosteroid that
modulates synaptic and extrasynaptic GABAA receptors.
We characterized pharmacokinetic (PK) and pharmacody-
namic (PD) properties of ALLO to support its use in sta-
tus epilepticus (SE). Our objectives were (1) assess the
adverse effects of intravenous (IV) ALLO at increasing
doses, ( 2 ) characterize its PK, and (3) develop a PK-PD
model. In our study, we included both healthy dogs $(n=3$ )
and those with spontaneous epilepsy ( $n=2$, 1 on pheno-
barbital [PB]). Single ALLO doses ranging between 1 and
$4 \mathrm{mg} / \mathrm{kg}$ were infused IV over 5 min. Serial blood samples
were collected up to 6 h. Plasma concentrations were
measured using a UPLC-MS/MS system. Non-
compartmental and compartmental PK analyses were per-
formed. Concentration-time profiles were best fit by a
two-compartment model (half-life: alpha $=1.7-3.2$ min,
beta $=13.2-39.3$ min). There was a dose-dependent in-
crease in ataxia and sedation. At 4 mg/kg, dogs were
immobile and briefly unarousable. Peak effects occurred
3 min into the infusion, with a return to baseline within
20 min. The dog on chronic PB had greater sedation at
2 mg/kg, which correlated with altered PK. In conclusion,
IV ALLO exhibited a rapid central nervous system PD
effect. When infused over 5 min, it was well-tolerated
up to 2 mg $/ \mathrm{kg}$ in dogs with and without epilepsy.
Different dosing regimens may be warranted in dogs on PB. Our results support further evaluation of ALLO for SE treatment in dogs and humans.

Title: Detection of Memory and Cognitive Impairment in Type II Diabetic Patients: A Comparison of MemTrax, AD8 and MMSE in an Old Chinese Population

Authors: Guoqiang Xing, PhD, The Second Clinical College of North Sichuan Medical College, Nanchong Central Hospital, Liang Zhou ${ }^{1,2}$, Qi Zhang ${ }^{3}$, Mengjie Cai1, Xi Bai ${ }^{1}$, Zhiwei Guo ${ }^{1}$, Qiwen $\mathrm{Mu}^{1}$, Han Zhang ${ }^{1}$, Yuzhang Zhao, Xuezhu Huang ${ }^{1}$, Mingming Ding ${ }^{3}$, John S. Lin ${ }^{3}$, Xianbo Zhou $^{4}$, and J. Wesson Ashford ${ }^{5,6},{ }^{1}$ Department of Radiology \& Imaging, Institute of Rehabilitation and Development of Brain Function, The Second Clinical College of North Sichuan Medical College, Nanchong Central Hospital, Nanchong, China; ${ }^{2}$ The Clinical Medical College of Southwest Medical University, Luzhou, Sichuan, China; ${ }^{3}$ SJN Biomed LTD, Kunming, China; ${ }^{4}$ Center for Alzheimer Research, Washington Institute of Clinical Research, Vienna, VA; ${ }^{5}$ War Related Illness and Injury Study Center, VA Palo Alto Health Care System, Palo Alto, CA; ${ }^{6}$ Department of Psychiatry and Behavioral Sciences, Stanford University School of Medicine, Stanford, CA

Abstract: Type 2 diabetes mellitus (T2DM) is a known risk factor for mild cognitive impairment and dementia. Early detection of memory and cognitive impairment in T2DM patients could afford better care and disease management. However, we are not aware of a quick, easy and preferably self-administered test to screen for memory and cognitive impairment in these patients. In this study, we have compared three different tests: an online based MemTrax memory test which is based on the continuous recognition paradigm, the Ascertain Dementia 8 (AD8) questionnaire and Mini-Mental State Examination (MMSE) in 164 T2DM patients (72 males, 92 females) in a community in Nanchong, Sichuan China. MemTrax memory takes about 2 min to administer and can be used either in a doctor's office or anywhere that is relatively quiet. Our screening team (nine people) examined about 40 to 50 T2DM patients every morning for 5 days. Preliminary analyses indicate MemTrax memory could be a valuable tool for the detection of memory impairment in T2DM patients. If the final analyses warrantee the use of MemTrax memory test for early detection of memory and cognitive impairment in T2DM patients, we intend to screen most of the 100,000 old adults identified to be at risk for cardiovascular and CNS diseases including 20,000 diabetic patients identified during the last 5 years of physical examinations by our Nanchong Chronic Disease Management Platform. A total of 20,000 plus blood samples have been collected from these patients. 
We intend to perform genome sequencing and APOE genotyping of these samples in collaboration with appropriate partners. In conclusion, compared to AD8 and MMSE, MemTrax is a more effective tool for rapid screening of a large population of diabetic patients at risk of memory and cognitive impairment.

Title: Valproic Acid Derivatives Valnoctamide and Secbutylpropylacetamide Terminate Tetramethylenedisulfotetramine Induced Status Epilepticus More Rapidly Than Diazepam

Authors: Dorota Zolkowska, Department of Neurology, School of Medicine, University of California, Davis, Meir Bialer $^{2}$; Carson W. Flamm ${ }^{1}$; Nancy Garibay ${ }^{1}$; David Bibi ${ }^{2}$; Michael A. Rogawski ${ }^{1}$.

${ }^{1}$ Department of Neurology, School of Medicine, University of California, Davis, Sacramento, CA, United States; ${ }^{2}$ Pharmaceutics Institute for Drug Research, School of Pharmacy, Ein Karem, Faculty of Medicine, The Hebrew University of Jerusalem, Jerusalem, Israel.

Abstract: Valnoctamide (VCD) and sec-butylpropylacetamide (SPD) are amide derivatives of valproic acid that protect against seizures and terminate ongoing status epilepticus (SE) in rodent models. Tetramethylenedisulfotetramine (TETS) is a highly lethal neurotoxic rodenticide that acts as a noncompetitive GABAA receptor antagonist and is considered a chemical threat agent. Severe TETS intoxication in humans leads to refractory convulsive SE. Here, we used a mouse TETS SE model to compare the efficacy of VCD and SPD with diazepam (DZ), a standard treatment for acute seizures. Mice received a single dose of riluzole $(10 \mathrm{mg} / \mathrm{kg}$, IP) and $10 \mathrm{~min}$ later a lethal dose of TETS $(0.2 \mathrm{mg} / \mathrm{kg}$, IP). We recorded the latency to cessation of behavioral SE (the interval between the first myoclonic twitch and termination of seizure activity) and the incidence of survival $72 \mathrm{~h}$ post SE. SDP, VCD and DZ were injected IP at $40 \mathrm{~min}$ after the first myoclonic twitch. SPD at doses 54 and $100 \mathrm{mg} / \mathrm{kg}$ terminated SE within, respectively, 4 and 2 min and protected 65 and $100 \%$ of animals from lethality. VCD at doses 50 and $100 \mathrm{mg} / \mathrm{kg}$ terminated SE within $\sim 7$ and $\sim 2$ min and protected 62.5 and $90 \%$ of animals from lethality. DZ at a dose of $1.8 \mathrm{mg} / \mathrm{kg}$ terminated SE in within $13 \mathrm{~min}$ in $91 \%$ of animals and protected $89.2 \%$ of animals from lethality. All drugs induced sedation at the doses used. SPD and VCD at the doses tested more rapidly and more reliably terminated SE than DZ but all three drugs protected animals from lethality. Support: NINDS grant \#1U54NS079202 (DZ, MAR); UC Davis CAMP Program (NG). 